\title{
The Adhesion Mechanism of Marine Mussel Foot Protein: Adsorption of L-Dopa on $\alpha$ - and $\beta$-Cristobalite Silica Using Density Functional Theory
}

\author{
Shabeer Ahmad Mian and Younas Khan \\ Department of Physics, University of Peshawar, Peshawar, Pakistan \\ Correspondence should be addressed to Shabeer Ahmad Mian; shabeerahmad@upesh.edu.pk
}

Received 28 August 2016; Revised 20 November 2016; Accepted 21 December 2016; Published 15 January 2017

Academic Editor: Anton Kokalj

Copyright (C) 2017 S. A. Mian and Y. Khan. This is an open access article distributed under the Creative Commons Attribution License, which permits unrestricted use, distribution, and reproduction in any medium, provided the original work is properly cited.

\begin{abstract}
Marine mussels strongly adhere to various surfaces and endure their attachment under a variety of conditions. In order to understand the basic mechanism involved, we study the adsorption of L-dopa molecule on hydrophilic geminal and terminal isolated silanols of silica (001) surface. High content of modified amino acid L-dopa is found in the glue-like material secreted by the mussels through which it sticks to various surfaces under water. To understand the adsorption behavior, we have made use of periodic Density Functional Theory (DFT) study. The L-dopa molecule adheres to silica surfaces terminated with geminal and terminal silanols via its catechol part. In both cases, the adhesion is achieved through the formation of $4 \mathrm{H}$-bonds. A binding energy of 29.48 and $31.67 \mathrm{kcal} / \mathrm{mol}$ has been estimated, after the inclusion of dispersion energy, for geminal and terminal silanols of silica, respectively. These results suggest a relatively stronger adhesion of dopa molecule for surface with terminal isolated silanols.
\end{abstract}

\section{Introduction}

Mussels are marine organisms that have the ability to attach themselves firmly to a variety of surfaces including glass, plastic, and metal oxides [1]. Their adhesion is so strong that they can even stick to nonstick materials such as Teflon [2]. Furthermore, these mussels are capable of achieving adhesion in a variety of conditions such as wet, dry, and salty environments [3-5]. Mussels are usually found in rocky, wind-swept, and wave-swept seacoasts. Their adhesion should be rapid and strong; otherwise they may be dislodged by the energetic incoming waves. Mussels secrete a glue-like sticky material, known as byssus, which is responsible for the strong adhesion to rocks and other surfaces in turbulent marine environment [6]. The byssus is a bundle of threadlike materials that spreads out in a radially outward direction. It consists of four parts, namely, plaque, thread, stem, and root [7]. Plaque is that part of the byssal thread which is attached with the foreign surface, whereas the stem is connected with tissues of the organism by means of the root. Stem and plaque are attached with each other through the thread. Mussel byssus is proteinaceous and does not have living cells. Byssal threads are attached to the root at the base of mussel foot where a combination of 12 retractor muscles controls the tension in them. During adhesion, the mussel foot emerges from the shell, finds suitable surface, and becomes almost motionless after the adhesion. Field tests have revealed significant information about the tenacity (ability to resist dislodgement as a result of external force) of mussels $[8,9]$. The tenacity of an individual California mussel (Mytilus californianus) is $300 \mathrm{~N}$ in lift mode and $180 \mathrm{~N}$ in parallel displacement mode. Since a mussel has $50-100$ byssal threads at a time and all the threads contribute to load bearing in lift mode only, the per-thread tenacity of California mussel has a maximum value of approximately $6 \mathrm{~N}$. More than 25 different mussel foot proteins ( $\mathrm{mfp}$ ) have been identified in byssus, out of which 5 (mfp-2 to mfp-6) are unique to plaque [10]. These $5 \mathrm{mfp}$ have a high content of the usually rare modified amino acid 3,4-dihydroxy-L-phenylalanine (Ldopa). It is widely believed that it is the catechol functionality of L-dopa molecule that gets attached with external surface during the adhesion process [11-15]. Mian et al. demonstrated 
that the catechol part of dopa interacts with the surface in dry conditions [16] and displaces the preadsorbed water molecules to bind with hydrophilic silica surface [16-18]. By using an AFM, researchers have investigated the single molecule mechanics of mussel adhesion by measuring the force of a single dopa molecule in contact with a wet $\mathrm{TiO}_{2}$ surface [19]. Bahri et al. performed the adsorption and surface complexation investigation of L-dopa on Rutile surface in $\mathrm{NaCl}$ solution. They reported that L-dopa forms two species on the surface, with their proportion strongly depending on the $\mathrm{pH}[20]$.

Understanding the basic principles underlying the adhesion of mussels may lead to useful applications in a variety of fields, especially in medicine, dentistry, surface coatings, biomedical engineering, and bionanotechnology. There have been efforts to design biomimetic adhesives that are efficient, nontoxic, and biocompatible. In recent years, a number of scientific studies have been dedicated to design catecholfunctionalized copolymers; for example, Messersmith et al. have created tough, nonswelling bioadhesives based on catechol-modified polymers [21]. Mussel inspired bioadhesives have recently been used in cancer drug delivery and removal of infected tumors. Gold nanorod surface has been coated with mussel inspired bioadhesives. When irradiated, the nanorods produce localized heat which destroys the cancer cells [22]. Researchers have recently reported injectable synthetic polymers inspired by mussel adhesives. These citrate-based adhesives are low-cost, nontoxic, and nonallergic [23].

In this study we will simulate the adsorption behavior of L-dopa molecule on hydroxylated, hydrophilic surfaces of silica having terminal isolated and geminal silanols. In order to understand the atomic level details of adsorption phenomenon, we will use DFT energy calculations and DFT based molecular dynamics simulation. We will consider two types of silica surfaces, namely, silica surface with geminal and terminal isolated silanols. Geminal silica is the one which is terminated with two $\mathrm{OH}$ ions attached with each surface silicon atom whereas terminal silanols are single $\mathrm{OH}$ ions attached with each surface silicon. In this study, we have chosen the (001) surface of hydroxylated $\alpha$ - $\beta$-cristobalite silica to model amorphous silica because both materials have almost equal densities and refractive indices [15-18]. The present silica has a silanol density of 8.1 per $\mathrm{nm}^{2}$ which covers the silanols density of amorphous silica with a value of 5 hydroxyls per $\mathrm{nm}^{2}$.

\section{Computational Method}

We performed ab initio molecular dynamics and energy calculations using version 3.2 of the SIESTA code [24]. In this study, we have used the generalized gradient approximation (GGA) as the exchange and corelation functional, by the application of revised Purdue-Burke-Enzerhof (RPBE) method [25]. Norm-conserving pseudopotentials proposed by Troullier and Martins [26] were used with standard double zeta potential (DZP) basis set. Using the Monkhorst-Pack scheme [27], Brillouin zone sampling was performed with 3 $\times 3 \times 1 k$-points. The geometries were optimized separately before and the L-dopa molecule was placed $3 \AA$ above the surface to perform DFT calculations. All the calculations were carried out using the conjugate gradient (CG) method [28], and cell volume was allowed to vary. The optimization was performed until the atomic forces reached a value less than $(0.04 \mathrm{eV} / \AA)$. A mesh cut-off [29] of $2.72 \mathrm{keV}$ was used for our atomic orbitals. No plane waves were used during the calculations.

The initial configuration for optimization of bulk $\alpha-\beta$ cristobalite was taken from the rectangular unit cell with lattice parameters of 4.97 and $6.93 \AA$, having the $P 4121$ symmetry [30]. Initial geometry of both silica surfaces was constructed using the following steps: we considered a slab of bulk $\alpha$ - $\beta$-cristobalite having 16 atomic layers along the $z$-direction. The slab consisted of $3 \times 3$ layers in the plane normal to the surface that is the $x-y$ plane, when viewed from the top. The top silica surface was obtained by cleaving the bulk system along the (001) direction. The simulation box which we considered had dimensions of $A=B=$ $14.7 \AA$ and $C=40 \AA$. A vacuum gap of sufficient length was introduced along the vertical direction to remove the periodicity. During the optimization, the bottom Si atoms were kept under constraint. The number of atoms in geminal silica was 198, whereas L-dopa consisted of 25 atoms, resulting in a total of 223 atoms in our system.

Using the following relation, binding energy of the system was calculated:

$$
\Delta E=-\left[E_{D S}^{d+s}(D S)-E_{D}^{d}(D)-E_{S}^{s}(S)+\delta_{\mathrm{BSSE}}\right] .
$$

Throughout this text we have used a notation system in which the term $E_{X}^{Y}(Z)$ represents energy of the system $Z$ in the geometry of $X$ using the basis set $Y$. In (1), $E_{D S}^{d+s}(D S)$ represents the energy of molecule-surface complex, $E_{D}^{d}(D)$ is the energy of the molecule (L-dopa), and $E_{S}^{s}(S)$ denotes energy of the surface $S$. Keep in mind that, in our definition of the binding energy, a positive sign shows attraction between the surface and the molecule. We applied the counterpoise method [31,32] to calculate the basis set superposition error using the equation:

$$
\delta_{\mathrm{BSSE}}=\left[E_{D S}^{d}(D)-E_{D S}^{d+s}(D)\right]+\left[E_{D S}^{s}(S)-E_{D S}^{d+s}(S)\right] .
$$

In (2), molecular and surface geometries are taken from their geometries in the molecule-surface complex. We have calculated $E_{M D}^{d}(D)$ using the molecular basis and $E_{M S}^{s}(S)$ using the basis of the surface. $E_{M S}^{m+s}(M)$ and $E_{M S}^{m+s}(S)$ have been calculated using the basis for molecule-surface complex.

Traditional DFT methods are generally used for the description of H-bond; however, it does not include dispersion interaction between the atoms. In this study, we determined the dispersion interaction using the method of Grimme et al. [33, 34].

\section{Results and Discussion}

3.1. Optimized Geometry of L-Dopa. The adhesive and cohesive properties of mussels have been attributed to the presence of high amount (up to $30 \mathrm{~mol} \%$ ) of L-dopa in the 
TABLE 1: Structural parameters of optimized catechol.

\begin{tabular}{lcccccccccc}
\hline & $d_{1}(\AA)$ & $d_{2}(\AA)$ & $d_{1}^{\prime}(\AA)$ & $d_{2}^{\prime}(\AA)$ & $\theta_{1}\left(^{\circ}\right)$ & $\theta_{2}\left(^{\circ}\right)$ & $\theta_{1}^{\prime}\left(^{\circ}\right)$ & $\theta_{2}^{\prime}\left(^{\circ}\right)$ & $\phi_{1}\left(^{\circ}\right)$ & $\phi_{2}\left(^{\circ}\right)$ \\
\hline Isolated & 1.35 & 0.97 & 0.98 & 1.38 & 120.7 & 113.5 & 107.1 & 111.3 & -1.3 & 1.6 \\
Isolated $^{\mathrm{a}}$ & 1.36 & 0.97 & 0.98 & 1.38 & 120.7 & 113.4 & 107.1 & 111.3 & -1.3 & 1.6 \\
\hline
\end{tabular}

${ }^{\mathrm{a}}$ Previous calculation by Mian et al. [16].

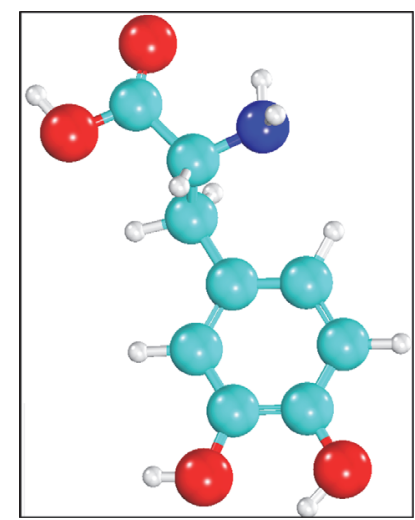

(a)

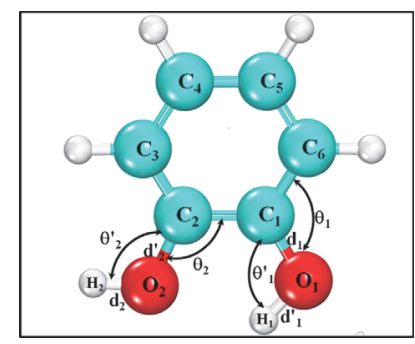

(b)

FIGURE 1: Optimized geometry of L-dopa (a) and its catechol functionality (b).

plaque/substrate interface. L-dopa, a usually rare catecholic amino acid, is formed as a result of posttranslational modification of tyrosine. Figure 1 shows the optimized geometry of dopa (a) and its catechol group (b). The catechol part has been labelled to indicate various bond lengths and angles. The structural parameters of dopa have been summarized in Table 1.

3.2. Adsorption Geometry of L-Dopa On Silica Surface. As a first step, we designed the initial geometries of L-dopa and silica. These geometries were separately optimized until the forces between their atoms were less than a certain threshold value. The optimized structure of L-dopa was placed at a distance of $3 \AA$ above both surfaces that is silica with geminal and terminal isolated silanols, and the combined geometry was once again relaxed. The resultant geometries, which show the adsorption behavior of L-dopa, are shown in Figures 2 and 3 .

Figure 2 shows the side and top view of L-dopa adsorbed on geminal silica. For visual clarity only the top-most layer of silica has been shown as ball and stick. The siloxane bridges attached to the silanols have been shown as lines. The figure shows that the two $\mathrm{OH}$ groups of the catechol part of L-dopa form 4 hydrogen bonds with the surface (represented by black dashed lines). It should be noted that the L-dopa molecule acts as a donor as well as acceptor of $\mathrm{H}$ to form the hydrogen bonds.

Figure 3 represents side view and top view of the adsorption behavior of L-dopa molecule on silica surface having terminal silanols. As expected and similar to the case of geminal surface, the two $\mathrm{OH}$ groups of catechol ring of the molecule form 4 hydrogen bond with the surface. Here again the $\mathrm{OH}$ groups act as donors as well as acceptors of $\mathrm{H}$ to form the bonds. The fact that catechol part of L-dopa is responsible for its strong adhesion with the silica surface is in agreement with previously reported findings [15-18]. In these studies, the catechol part of L-dopa molecule was adsorbed on silica surface under dry and wet conditions and similar behavior was observed as we have reported here.

3.3. Binding Energy and BSSE Calculations. Using (1) and (2), respectively, the binding energy, basis set superposition error (BSSE), and dispersion energy were calculated for the dopasilica system. The result has been summarized in Table 2 for both surfaces that is silica with geminal and terminal isolated silanols. In the case of geminal silica, a binding energy of 


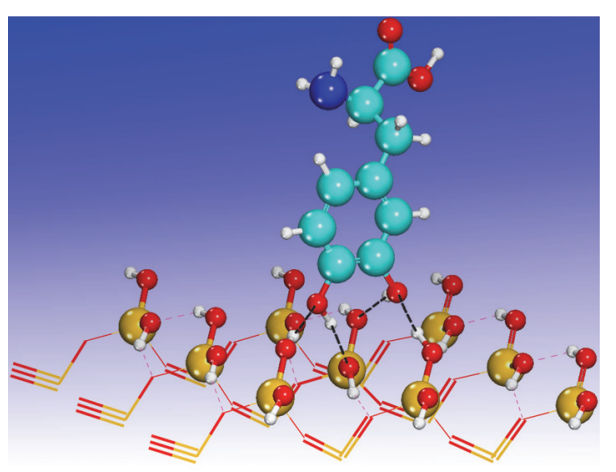

(a)

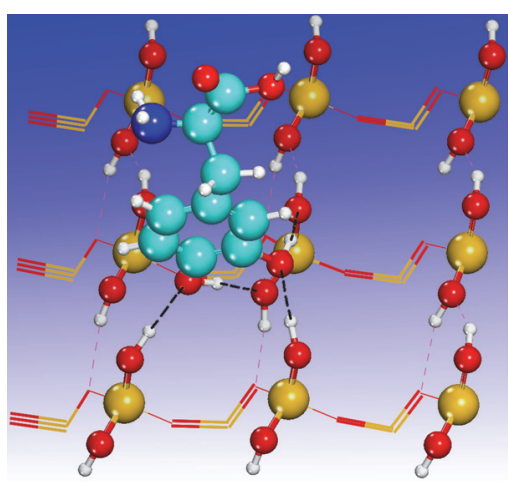

(b)

FIGURE 2: Side view (a) and top view (b) of dopa molecule adsorbed on geminal silica surface with intermolecular distances of $1.85 \AA$.

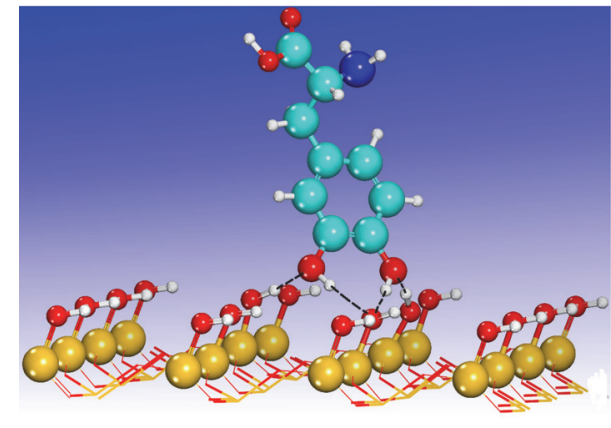

(a)

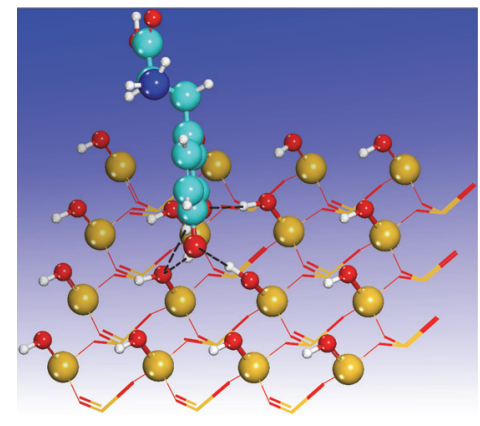

(b)

Figure 3: Side view (a) and top view (b) of L-dopa adsorption geometry on silica surface with intermolecular distances of $1.87 \AA$ making four hydrogen bonds with terminal isolated silanols.

TABLE 2: Binding energy $(\Delta E)(\mathrm{kcal} / \mathrm{mol})$ of L-dopa adsorbed on silica surfaces.

\begin{tabular}{lcc}
\hline Energy & Silica (001) & Silica (111) \\
\hline$\Delta E$ & $9.8(25.9)$ & $11.5(26.49)$ \\
$\Delta E_{\text {VWdisp }}$ & 29.5 & 31.7 \\
\hline
\end{tabular}

${ }^{*}$ BSSE correction for each binding energy ( $\delta$ BSSE) appears in parentheses.

$9.83 \mathrm{kcal} / \mathrm{mol}$ has been calculated for the L-dopa molecule. In order to get the average binding energy per hydrogen bond, we divided this value by the total number of hydrogen bonds, which resulted in a value of $2.46 \mathrm{kcal} / \mathrm{mol}$. This value is in the typical range of binding energy of a hydrogen bond [35]. A BSSE value of $25.90 \mathrm{kcal} / \mathrm{mol}$ was estimated which is $72.5 \%$ of the uncorrected energy. In the case of terminal silanols, a binding energy of $11.45 \mathrm{kcal} / \mathrm{mol}$ was estimated. The average binding energy per hydrogen bond in this case is $2.86 \mathrm{kcal} / \mathrm{mol}$ and a BSSE value of $26.49 \mathrm{kcal} / \mathrm{mol}$ has been calculated approximately $69.84 \%$ of the uncorrected binding energy. A large basis set superposition error has been reported in several Density Functional Theory calculations close to our calculated value that is the C60 adsorption on silicon (001) surface using PBE-GGA DFT with DZP basis almost close to our calculations [36]. Also in the glycine adsorption on an edingtonite silica the BSSE exceeds relatively the uncorrected binding energy value [37]. Regarding the binding energy $(\Delta E)$ calculation for ammonia $\left(\mathrm{NH}_{3}\right)$ on silica surface, Civalleri and Ugliengo [38] reported that the BSSE of a periodic calculation is larger.

The RPBE functional which we have used in this calculation is accurate in the description of hydrogen bond $[39,40]$ but it does not take into consideration the dispersion interaction among the atoms. Using B3LYP-D2* for the adsorption of benzene-1,4-diol on hydroxylated silica, Rimola et al. reported that the dispersion energy term may be significant as it reaches up to $16.7 \mathrm{kcal} / \mathrm{mol}[41,42]$ in their calculations. In present calculations a method proposed by Grimme [33] has been used to estimate the dispersion energy. A binding energy value of $19.65 \mathrm{kcal} / \mathrm{mol}$ has been calculated in the case of dopa molecule adsorbed on geminal silica (001). For silica (111) surface with terminal isolated silanols, a dispersion energy of $20.22 \mathrm{kcal} / \mathrm{mol}$ has been calculated. After adding the dispersion energy, the binding energy increased to $29.48 \mathrm{kcal} / \mathrm{mol}$ and $31.67 \mathrm{kcal} / \mathrm{mol}$ for surface with geminal and terminal isolated silanols, respectively. These final values of binding energy suggest that the adsorption of L-dopa molecule is stronger for surface with terminal silanols. It has been reported [41, 42] that Grimme's method generally overestimates binding energies, and to achieve a closer match 
with experiment a slight modification of this method is used [43].

\section{Conclusion}

Mussels are marine organisms that stick to a variety of surfaces under turbulent conditions. This strong adhesion is achieved through the secretion of a glue-like material called byssus. Mussel byssus is rich in the unusually rare Ldopa molecules. It is believed that the catecholic ring of the dopa molecule interacts with the surface to achieve adhesion. To understand the mechanism behind the phenomenon of mussel adhesion, we have used periodic Density Functional Theory (DFT). We simulated the adsorption of a single L-dopa molecule on silica surfaces having geminal and terminal silanols. The dopa molecule interacts with both surfaces through its catechol group. The catecholic ring of the molecule forms 4 hydrogen bonds and acts as a donor as well as an acceptor of hydrogen to form the bonds. The binding energy in the case of geminal silanols was estimated to be $9.83 \mathrm{kcal} / \mathrm{mol}$ which augmented to $29.48 \mathrm{kcal} / \mathrm{mol}$ when the dispersion energy term was added to it. For silica surface having terminal silanols, we calculated a binding energy of $11.5 \mathrm{kcal} / \mathrm{mol}$ which increased to $31.67 \mathrm{kcal} / \mathrm{mol}$ with the inclusion of dispersion energy. We can conclude that the catechol group indeed plays a pivotal role when dopa interacts with a silica surface. The adsorption of L-dopa molecule on both surfaces through the surface silanols is indistinguishable in terms of binding energy.

\section{Competing Interests}

The authors declare that there is no conflict of interests regarding the publication of this paper.

\section{Acknowledgments}

Shabeer Ahmad Mian, lab members, and coworkers are very thankful to the GIKI Super Computer Lab especially to Dr. Masroor Hussain and his team for providing his lab facility to perform this work.

\section{References}

[1] Q. Lu, E. Danner, J. H. Waite, J. N. Israelachvili, H. Zeng, and D. S. Hwang, "Adhesion of mussel foot proteins to different substrate surfaces," Journal of the Royal Society Interface, vol. 10, no. 79, pp. 1-11, 2013.

[2] H. Lee, S. M. Dellatore, W. M. Miller, and P. B. Messersmith, "Mussel-inspired surface chemistry for multifunctional coatings," Science, vol. 318, no. 5849, pp. 426-430, 2007.

[3] J. H. Waite, "Nature's underwater adhesive specialist," International Journal of Adhesion and Adhesives, vol. 7, no. 1, pp. 9-14, 1987.

[4] H. Lee, B. P. Lee, and P. B. Messersmith, "A reversible wet/dry adhesive inspired by mussels and geckos," Nature, vol. 448, no. 7151, pp. 338-341, 2007.

[5] H. Zhao, N. B. Robertson, S. A. Jewhurst, and J. H. Waite, "Probing the adhesive footprints of Mytilus californianus byssus,"
Journal of Biological Chemistry, vol. 281, no. 16, pp. 11090-11096, 2006.

[6] N. K. Kaushik, N. Kaushik, S. Pardeshi, J. G. Sharma, S. H. Lee, and E. H. Choi, "Biomedical and clinical importance of musselinspired polymers and materials," Marine Drugs, vol. 13, no. 11, pp. 6792-6817, 2015.

[7] B. P. Lee, P. B. Messersmith, J. N. Israelachvili, and J. H. Waite, "Mussel-inspired adhesives and coatings," Annual Review of Materials Research, vol. 41, pp. 99-132, 2011.

[8] E. C. Bell and J. M. Gosline, "Mechanical design of mussel byssus: material yield enhances attachment strength," Journal of Experimental Biology, vol. 199, no. 4, pp. 1005-1017, 1996.

[9] E. C. Bell and J. M. Gosline, "Strategies for life in flow: tenacity, morphometry, and probability of dislodgment of two Mytilus species," Marine Ecology Progress Series, vol. 159, pp. 197-208, 1997.

[10] J. H. Waite, H. C. Lichtenegger, G. D. Stucky, and P. Hansma, "Exploring molecular and mechanical gradients in structural bioscaffolds," Biochemistry, vol. 43, no. 24, pp. 7653-7662, 2004.

[11] M. Yu, J. Hwang, and T. J. Deming, "Role of L-3,4dihydroxyphenylalanine in mussel adhesive proteins," Journal of the American Chemical Society, vol. 121, no. 24, pp. 5825-5826, 1999.

[12] S.-K. Chen, B.-C. Wang, T.-G. Zhou, Y.-Z. Feng, H.-M. Liang, and W.-Z. Huang, "Theoretical investigation on the reaction of adhesion unit dopa in mussel with electrolyzing seawater," Colloids and Surfaces B: Biointerfaces, vol. 70, no. 2, pp. 243-247, 2009.

[13] P. A. Brooksby, D. R. Schiel, and A. D. Abell, "Electrochemistry of catechol terminated monolayers with $\mathrm{Cu}(\mathrm{II}), \mathrm{Ni}(\mathrm{II})$ and Fe(III) cations: a model for the marine adhesive interface," Langmuir, vol. 24, no. 16, pp. 9074-9081, 2008.

[14] H. G. Silverman and F. F. Roberto, "Understanding marine mussel adhesion," Marine Biotechnology, vol. 9, no. 6, pp. 661681, 2007.

[15] K. E. Adraa, V. Timon, J.-F. Lambert et al., "Adsorption of 1DOPA intercalated in hydrated Na-saponite clay: a combined experimental and theoretical study," Journal of Physical Chemistry C, vol. 116, no. 50, pp. 26414-26421, 2012.

[16] S. A. Mian, L. C. Saha, J. Jang, L. Wang, X. Gao, and S. Nagase, "Density functional theory study of catechol adhesion on silica surfaces," Journal of Physical Chemistry C, vol. 114, no. 48, pp. 20793-20800, 2010.

[17] S. A. Mian, X. Gao, S. Nagase, and J. Jang, "Adsorption of catechol on a wet silica surface: density functional theory study," Theoretical Chemistry Accounts, vol. 130, no. 2-3, pp. 333-339, 2011.

[18] S. A. Mian, L.-M. Yang, L. C. Saha, E. Ahmed, M. Ajmal, and E. Ganz, "A fundamental understanding of catechol and water adsorption on a hydrophilic silica surface: exploring the underwater adhesion mechanism of mussels on an atomic scale," Langmuir, vol. 30, no. 23, pp. 6906-6914, 2014.

[19] H. Lee, N. F. Scherer, and P. B. Messersmith, "Single-molecule mechanics of mussel adhesion," Proceedings of the National Academy of Sciences of the United States of America, vol. 103, no. 35, pp. 12999-13003, 2006.

[20] S. Bahri, C. M. Jonsson, C. L. Jonsson, D. Azzolini, D. A. Sverjensky, and R. M. Hazen, "Adsorption and surface complexation study of L-DOPA on rutile $\left(\alpha-\mathrm{TiO}_{2}\right)$ in $\mathrm{NaCl}$ solutions," Environmental Science \& Technology, vol. 45, no. 9, pp. 39593966, 2011. 
[21] D. G. Barrett, G. G. Bushnell, and P. B. Messersmith, "Mechanically robust, negative-swelling, mussel-inspired tissue adhesives," Advanced Healthcare Materials, vol. 2, no. 5, pp. 745-755, 2013.

[22] J. Su, F. Chen, V. L. Cryns, and P. B. Messersmith, "Catechol polymers for $\mathrm{pH}$-responsive, targeted drug delivery to cancer cells," Journal of the American Chemical Society, vol. 133, no. 31, pp. 11850-11853, 2011.

[23] M. Mehdizadeh, H. Weng, D. Gyawali, L. Tang, and J. Yang, "Injectable citrate-based mussel-inspired tissue bioadhesives with high wet strength for sutureless wound closure," Biomaterials, vol. 33, no. 32, pp. 7972-7983, 2012.

[24] J. M. Soler, E. Artacho, J. D. Gale et al., "The SIESTA method for ab initio order-N materials simulation," Journal of Physics Condensed Matter, vol. 14, no. 11, pp. 2745-2779, 2002.

[25] B. Hammer, L. B. Hansen, and J. K. Nørskov, "Improved adsorption energetics within density-functional theory using revised Perdew-Burke-Ernzerhof functionals," Physical Review $B$, vol. 59, no. 11, pp. 7413-7421, 1999.

[26] N. Troullier and J. L. Martins, "Efficient pseudopotentials for plane-wave calculations," Physical Review B, vol. 43, no. 3, pp. 1993-2006, 1991.

[27] H. J. Monkhorst and J. D. Pack, "Special points for Brillouinzone integrations," Physical Review B, vol. 13, no. 12, pp. 51885192, 1976.

[28] M. R. Hestenes and E. Stiefel, "Methods of conjugate gradients for solving linear systems," Journal of Research of the National Bureau of Standards, vol. 49, no. 6, pp. 409-436, 1952.

[29] J. M. Soler, E. Artacho, J. D. Gale et al., "The SIESTA method for ab initio order-N materials simulation," Journal of Physics: Condensed Matter, vol. 14, no. 11, pp. 2745-2779, 2002.

[30] J. J. Pluth, J. V. Smith, and J. Faber Jr., "Crystal structure of low cristobalite at 10, 293, and $473 \mathrm{~K}$ : variation of framework geometry with temperature," Journal of Applied Physics, vol. 57, no. 4, pp. 1045-1049, 1985.

[31] S. S. Xantheas, "On the importance of the fragment relaxation energy terms in the estimation of the basis set superposition error correction to the intermolecular interaction energy," The Journal of Chemical Physics, vol. 104, no. 21, pp. 8821-8824, 1996.

[32] F. B. Van Duijneveldt, J. G. C. M. Van Duijneveldt-Van De Rijdt, and J. H. Van Lenthe, "State of the art in counterpoise theory," Chemical Reviews, vol. 94, no. 7, pp. 1873-1885, 1994.

[33] S. Grimme, "Semiempirical GGA-type density functional constructed with a long-range dispersion correction," Journal of Computational Chemistry, vol. 27, no. 15, pp. 1787-1799, 2006.

[34] W. Smith, C. W. Yong, and P. M. Rodger, "DL_POLY: application to molecular simulation," Molecular Simulation, vol. 28, no. 5, pp. 385-471, 2002.

[35] A. J. Kinloch, "The science of adhesion-part 1 surface and interfacial aspects," Journal of Materials Science, vol. 15, no. 9, pp. 2141-2166, 1980.

[36] C. Hobbs, L. Kantorovich, and J. D. Gale, "An ab initio study of $\mathrm{C}_{60}$ adsorption on the $\mathrm{Si}\left(\begin{array}{ll}0 & 0\end{array}\right)$ surface," Surface Science, vol. 591, no. 1-3, pp. 45-55, 2005.

[37] A. Rimola, M. Sodupe, S. Tosoni, B. Civalleri, and P. Ugliengo, "Interaction of glycine with isolated hydroxyl groups at the silica surface: first principles B3LYP periodic simulation," Langmuir, vol. 22, no. 15, pp. 6593-6604, 2006.

[38] B. Civalleri and P. Ugliengo, "First principles calculations of the adsorption of $\mathrm{NH} 3$ on a periodic model of the silica surface," Journal of Physical Chemistry B, vol. 104, no. 40, pp. 9491-9499, 2000.
[39] Y. Zhao and D. G. Truhlar, "The M06 suite of density functionals for main group thermochemistry, thermochemical kinetics, noncovalent interactions, excited states, and transition elements: two new functionals and systematic testing of four M06-class functionals and 12 other functionals," Theoretical Chemistry Accounts, vol. 120, no. 1, pp. 215-241, 2008.

[40] T. van der Wijst, C. F. Guerra, M. Swart, and F. M. Bickelhaupt, "Performance of various density functionals for the hydrogen bonds in DNA base pairs," Chemical Physics Letters, vol. 426, no. 4-6, pp. 415-421, 2006.

[41] A. Rimola, B. Civalleri, and P. Ugliengo, "Physisorption of aromatic organic contaminants at the surface of hydrophobic/hydrophilic silica geosorbents: a B3LYP-D modeling study," Physical Chemistry Chemical Physics, vol. 12, no. 24, pp. 63576366, 2010.

[42] B. Civalleri, C. M. Zicovich-Wilson, L. Valenzano, and P. Ugliengo, "B3LYP augmented with an empirical dispersion term (B3LYP-D*) as applied to molecular crystals," CrystEngComm, vol. 10, no. 4, pp. 405-410, 2008.

[43] P. Jurečka, J. Černý, P. Hobza, and D. R. Salahub, “Density functional theory augmented with an empirical dispersion term. Interaction energies and geometries of 80 noncovalent complexes compared with ab initio quantum mechanics calculations," Journal of Computational Chemistry, vol. 28, no. 2, pp. 555-569, 2007. 

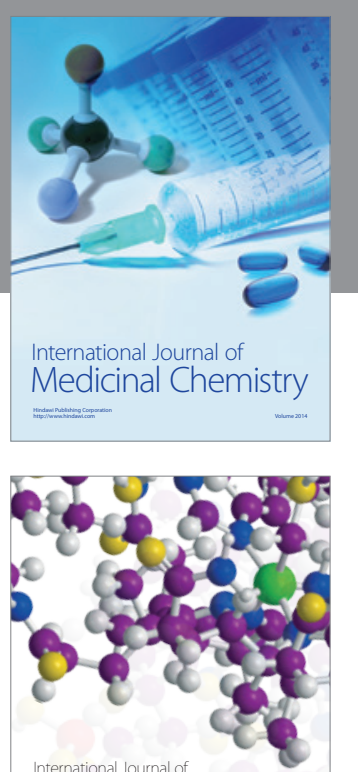

Carbohydrate Chemistry

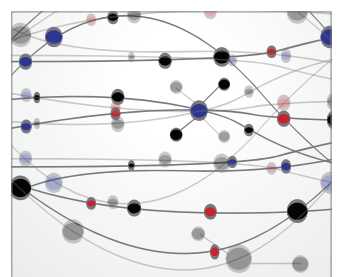

The Scientific World Journal
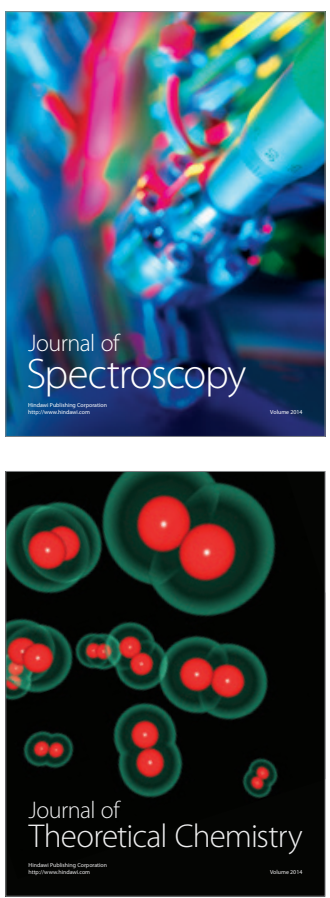
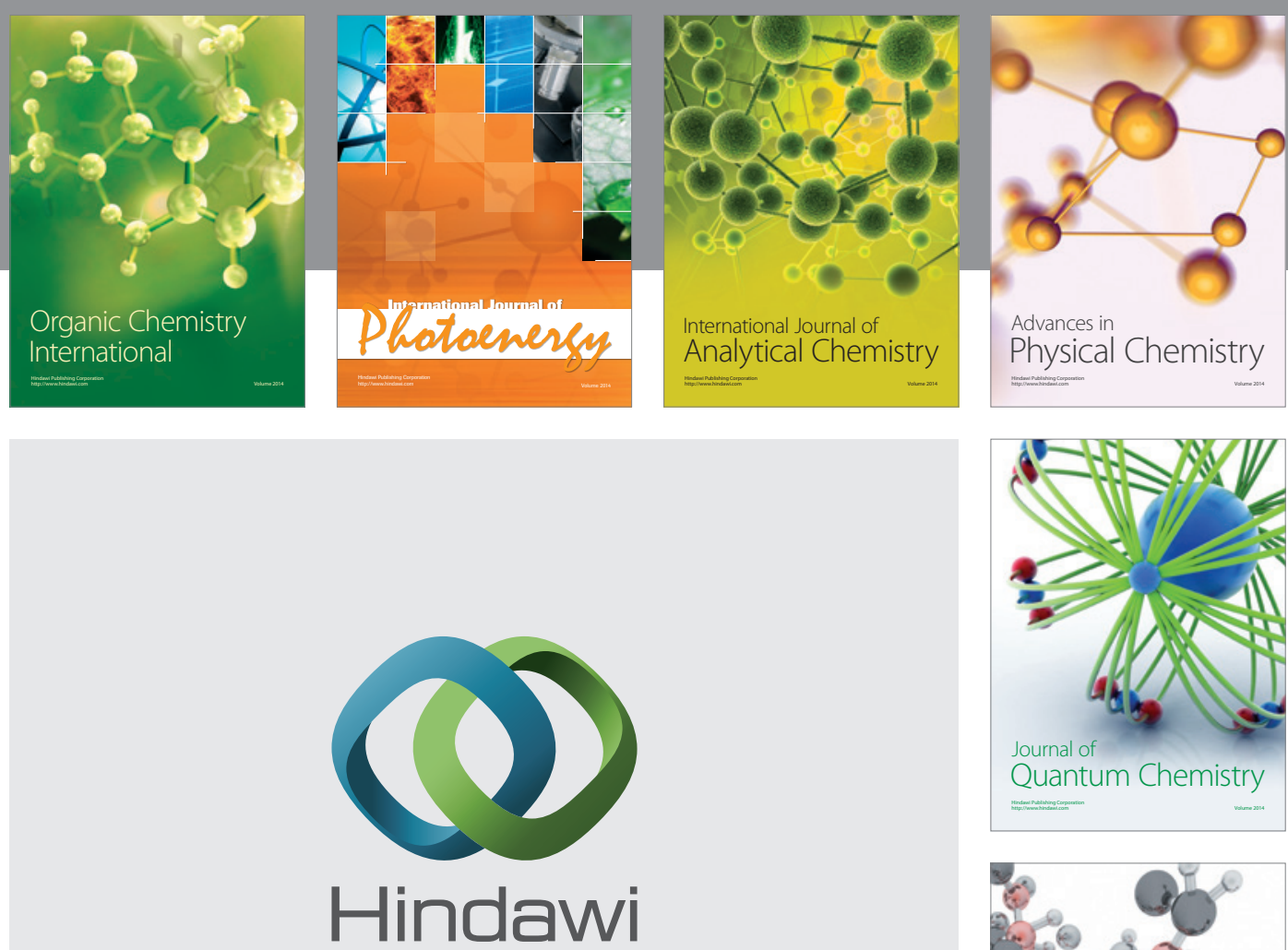

Submit your manuscripts at

https://www.hindawi.com

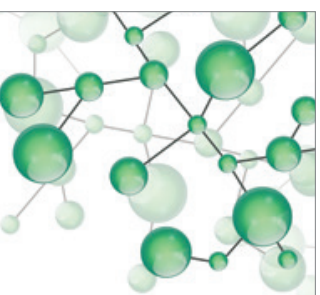

International Journal of

Inorganic Chemistry
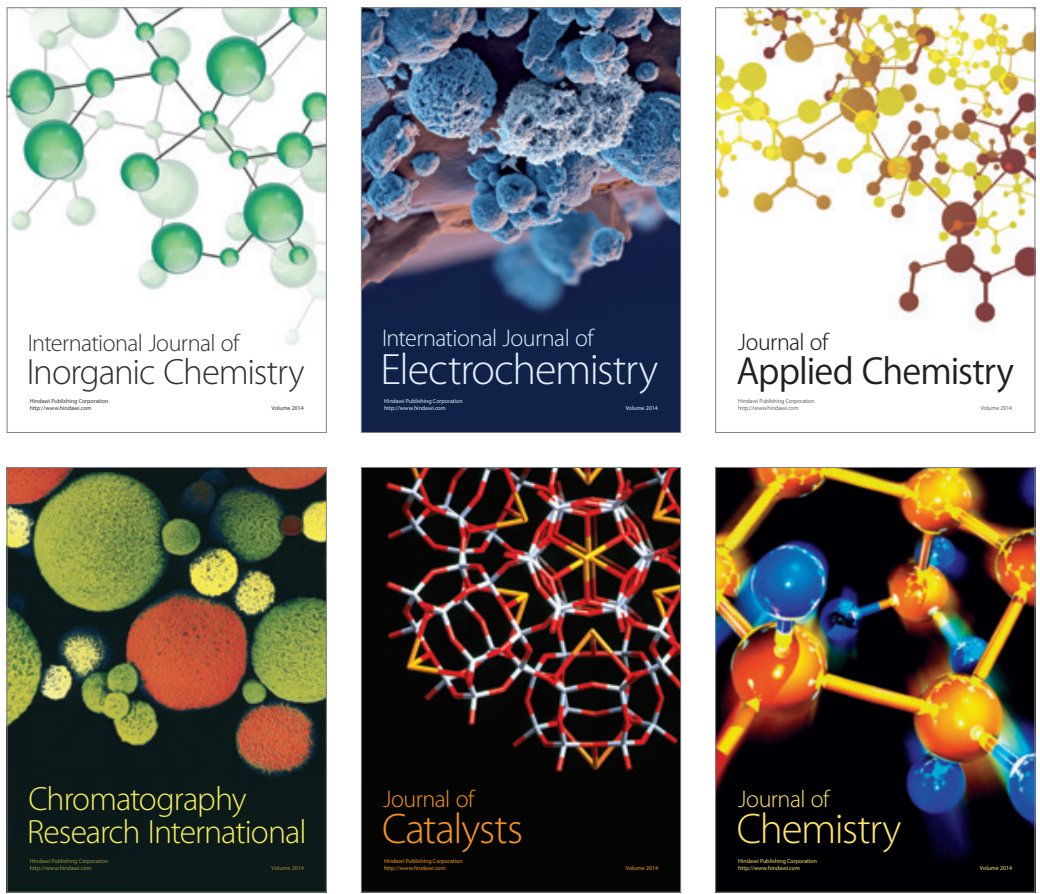

Journal of

Applied Chemistry
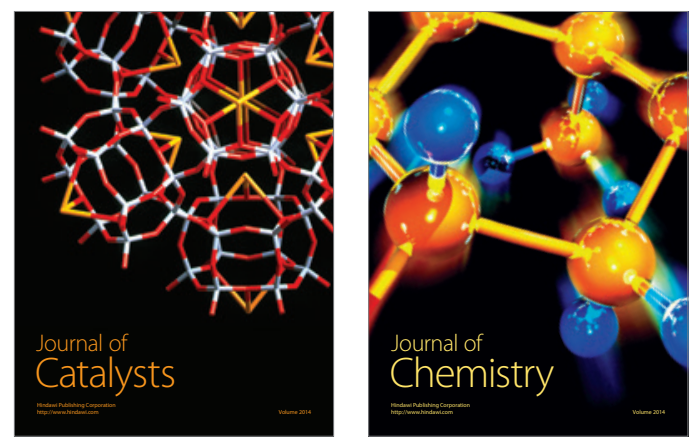
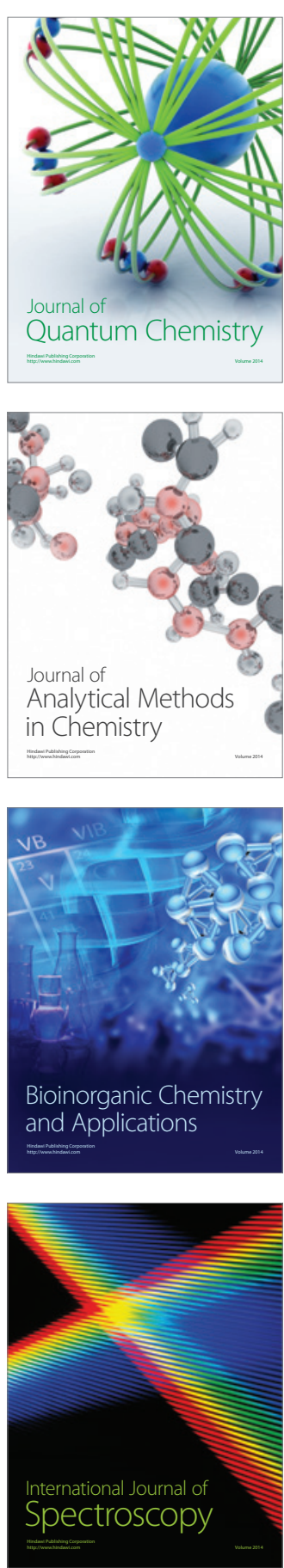